\title{
Transidentität aus medizinisch-ethischer Sicht
}

\author{
Christian Egarter
}

\section{Zusammenfassung}

Transidentität bzw. Gender-Inkongruenz geht mit einer Ablehnung der angeborenen biologischen Geschlechtsmerkmale und der damit verbundenen Geschlechtsidentität und -rolle einher. Die Prävalenz wird auf ca. 5,5 von 100.000 Individuen geschätzt. Als Ursache werden derzeit verschiedene veränderte Abschnitte des Gehirns angenommen, die strukturell und funktionell mit modernen Methoden untersucht wurden. Die Gesellschaft und insbesondere die moderne Medizin sollten überkommene pathologische Einstufungen überdenken, grundsätzlich die Selbsteinschätzung der Betroffenen ernst nehmen und generell das Wohl der Betroffenen bezüglich diagnostischer und therapeutischer Behandlung inklusive sexueller Empfindungsfähigkeit und Fertilität sowie sozialer und juristischer Aspekte in den Vordergrund stellen.

\section{Begriffsbestimmung: Intersexualität-Transidentität/Gender-Inkongruenz}

Intersexualität (im Englischen: Differences of Sex Development [DSD]) liegt vor, wenn ein Mensch genetisch und/oder anatomisch bzw. hormonell nicht eindeutig dem weiblichen oder männlichen Geschlecht zugeordnet werden kann. Davon unbedingt zu unterscheiden ist die Transidentität bzw. Gender-Inkongruenz, bei der sich ein Mensch mit dem zugewiesenen Geschlecht falsch oder unzureichend beschrieben fühlt oder grundsätzlich auch jede Form der Geschlechtszuweisung bzw. -kategorisierung ablehnt. Die bisher gebräuchlichen Begriffe "Transsexualität“ oder "Gender-Dysphorie“ unterliegen seit einiger Zeit der Kritik von Betroffenen und deren Selbsthilfegruppen. In der nächsten Version der WHO bezüglich der Klassifizierung („International Classification of Diseases“, ICD 11), die von der WHO im Mai 2019 verabschiedet wurde und am 1. Januar 2022 in Kraft treten soll, wurde dies berücksichtigt und der neutralere Begriff Gender-Inkongruenz eingeführt, der als ausgeprägte und persistierende Inkongruenz zwischen dem individuell erfahrenen und dem zugewiese- 
nen Geschlecht charakterisiert ist. Grundsätzlich will man damit erreichen, dass diese Situation nicht mehr als Persönlichkeits- oder Verhaltensstörung bzw. psychische Störung angesehen wird.

Die dänische Gesundheitsbehörde strich den Begriff „Transsexualität“ bereits seit Anfang des Jahres 2017 von der Liste psychischer Leiden. Damit gehört Dänemark weltweit neben Frankreich, wo Personen mit Gender-Inkongruenz seit 2010 nicht mehr als psychisch krank gelten, zu den Ausnahmen. Da generell aber die neue ICD-11-Version noch nicht gilt, werden hier weiter die medizinisch häufigen und leichter verständlichen Begriffe Intersexualität und Transidentität verwendet, die aber in keiner Weise pathologisierend oder abwertend gemeint sind. Weiter muss allerdings bedacht werden, dass die derzeitige Einstufung als Krankheit in den meisten Ländern noch den Betroffenen auch den Anspruch auf eine medizinische Behandlung sichert.

Der deutsche Arzt und Sexualforscher Magnus Hirschfeld prägte bereits 1923 für Menschen, die seelisch dem einen, körperlich aber dem anderen Geschlecht zugehören, den Begriff des „seelischen Transsexualismus“, einer geschlechtlichen Variation, die er als Vorstufe des Hermaphroditismus ansah. Heute wird diese dadurch definiert, dass die Geschlechtsidentifikation mit einer Ablehnung der biologischen und körperlichen Merkmale des angeborenen Geschlechts einhergeht, sowie durch die Zurückweisung der mit diesem verbundenen Rollenerwartungen sowie in vielen Fällen dem Verlangen, durch hormonelle und chirurgische Maßnahmen so weit wie möglich die körperliche Erscheinungsform des Identitätsgeschlechts anzunehmen und sozial bzw. juristisch im gewünschten Geschlecht zu leben. Gelegentlich wird allerdings auch jede Form der Geschlechtszuweisung und -kategorisierung abgelehnt. Das paradoxe Zugehörigkeitsgefühl führt meist im Laufe des Lebens zu zunehmend größerem Leidensdruck, der dann letztlich in vielen Fällen die Betroffenen veranlasst, ihr Äußeres entsprechend ihrer inneren Empfindung zu verändern bzw. dieser anzugleichen. Transidente Menschen können das Verlangen verspüren, ihren physischen, sozialen oder juristischen Status (bzw. Teile davon) zu verändern, um ihre Geschlechtsidentität damit in Einklang zu bringen. Von der Transidentität jedenfalls strikt abzugrenzen sind die verschiedenen Formen der Intersexualität (DSD) sowie die sexuelle Orientierung, die die sexuelle und emotionale Hinwendung von einer Person zu einer anderen mit ihren Aspekten der Hetero-, Homo- oder Bisexualität bezeichnet.

Bezüglich der Ursachen der Transidentität gibt es in den letzten Jahrzehnten einige Untersuchungen, die aber meist nur an wenigen Probanden durchgeführt wurden und teilweise unterschiedliche Ergebnisse liefer- 
ten. Die verfügbaren Daten von strukturellen und funktionellen Untersuchungen des Gehirns bei Transidentität scheinen in definierten Bereichen wie z. B. im Nucleus stria terminalis oder in Kernen des Hypothalamus (INAH3) eine Feminisierung bzw. Maskulinisierung oder dimorphe Strukturen zu zeigen, die sich teilweise unter hormoneller Behandlung weiter an das gewünschte Geschlecht anpassen (Smith et al. 2015). Somatostatin produzierende Neurone im Nucleus stria terminalis unterscheiden sich beispielsweise zunächst bei Frauen und Männern insofern, als Männer fast doppelt so viele Neurone aufweisen. Bei Personen mit Transidentität entspricht offenbar die Anzahl der Neurone dem subjektiven und nicht dem tatsächlichen Geschlecht und wird auch nicht durch die entsprechenden exogenen Sexualhormone beeinflusst (Kruijver et al. 2000).

Die Prävalenz der Transidentität nimmt in den letzten Jahren zu, was wahrscheinlich auf den mittlerweile in vielen westlichen Ländern eher liberaleren Umgang mit dieser Thematik zurückzuführen ist. Insgesamt schätzt man die Prävalenz auf etwa 5,5 von 100.000 Individuen, davon fallen 6,8 unter Mann-zu-Frau- (MzF) und 2,6 unter Frau-zu-MannTransidentität (FzM) (Meyer zu Hoberge 2009). Nicht-klinische Stichproben mit Fragebögen in größeren Kollektiven zeigen jedoch, dass eine - allerdings vom Ausmaß her sehr unterschiedliche - Ambivalenz gegenüber dem eigenen Geschlecht bei immerhin 4,6 Prozent der Männer und 3,2 Prozent der Frauen besteht (Kuyper 2012).

Beim Phänomen Transidentität geht es jedenfalls um einen besonders sensiblen und verletzbaren Bereich des Menschen, seine geschlechtliche Identität, die vom tatsächlichen Geschlecht und von den üblichen gesellschaftlichen Normvorstellungen abweicht, was immer wieder zu Diskriminierung von Betroffenen und zu einem gewissen Anpassungsdruck an die heterosexuelle Norm führen kann. Die Medizin und die Gesellschaft insgesamt stehen daher vor der Herausforderung, die subjektive Selbsteinschätzung Betroffener ernst zu nehmen, überkommene pathologische Einstufungen zu hinterfragen, grundsätzlich ein Recht auf Anderssein anzuerkennen und so zu einem neuen Verständnis dessen zu gelangen, was es in diesem Kontext bedeutet, das Recht der Betroffenen auf Sorge um ihre sexuelle Selbstbestimmung und Nichtdiskriminierung zu respektieren. Befragungen haben beispielsweise ergeben, dass Betroffene psychisch stärker unter sozialen Ausgrenzungen leiden als unter den direkten Folgen ihrer Transidentität.

In allen Überlegungen und Entscheidungen im Umgang mit Transidentität muss daher die bestmögliche Förderung des Wohls der Betroffenen im Vordergrund stehen. Dieses Wohl umfasst klarerweise nicht nur den medizinischen Erfolg von Maßnahmen, sondern die nachhaltige Zufrie- 
denheit Betroffener mit dem eigenen Körper und der eigenen Geschlechtsidentität. Es umfasst auch die Frage der sexuellen Empfindungsfähigkeit, die Möglichkeit, sexuelle Beziehungen eingehen zu können, sowie auch den Erhalt der Fortpflanzungsfähigkeit (Condat et al. 2018). Bei Transidentität erschließt sich das Wohl Betroffener nur unter Einbeziehung der Selbsterfahrung sowie der Meinung von Selbsthilfegruppen und Interessensverbänden.

\section{Diagnostische Evaluierung}

Die medizinische Diagnostik und der daraus resultierende Behandlungsprozess bei Transidentität sind relativ komplex und erfordern nicht nur die Koordination mehrerer Berufsgruppen, sondern üblicherweise auch die Langzeitbetreuung der betreffenden Personen. Deshalb erfolgt dieser Prozess heute meist in entsprechenden Kompetenzzentren, wo qualitativ hochwertige Betreuungs- und Behandlungsangebote zur Verfügung stehen.

Die Behandlungsstandards für Personen mit Transidentität wurden von der „World Association for Transgender Health" zusammengefasst (WPATH 2011; Coleman et al. 2012). Hier sind gewisse Rahmenbedingungen vorgegeben, die aber flexibel gestaltet sind, sodass die individuellen Bedürfnisse der transidenten Personen bedacht werden können. Um das Ziel der optimierten physischen und psychischen Anpassung zu erreichen, werden die betreffenden Personen zunächst zur grundsätzlichen Feststellung des Vorliegens einer Gender-Inkongruenz im Rahmen einer klinischpsychologischen, psychiatrischen, urologisch-gynäkologischen und/oder endokrinologischen Diagnostik durch entsprechende Experten evaluiert. Bei Vorliegen koexistenter psychiatrischer oder somatischer Störungen erfolgt meist die weiterführende Behandlung durch diesbezügliche Spezialisten.

Das Ergebnis der Evaluierung kann dann die formelle Diagnose Transidentität bzw. Gender-Inkongruenz sein und sollte vor allem andere psychiatrische Ursachen ausschließen. Manchmal zeigen transidente Personen aufgrund des gesellschaftlichen Umfelds eine chronische Stresssituation oder auch depressive Aspekte, Ängstlichkeit, Alkohol- und/oder DrogenAbusus, manchmal auch erhöhte Suizidraten oder Zwangs- bzw. Persönlichkeitsstörungen bis hin zum autistischen Formenkreis. Diese eventuell vorliegenden, koexistenten Veränderungen sollten entsprechend diagnostiziert und behandelt werden, da sie den Transitions-Prozess ungünstig beeinflussen können (Coleman et al. 2012). 
Eine besondere Herausforderung der letzten Zeit stellen Kinder und jugendliche Personen mit Gender-Inkongruenz dar. Es gibt derzeit noch keine klaren Zahlen zur Inzidenz; man geht in etwa von $<$ 1:2000 aus. Bei den meisten Betroffenen handelt es sich um eine passagere Erscheinung und nur bei einem kleinen Teil persistiert die Gender-Inkongruenz und es entwickelt sich ein entsprechender Leidensdruck. Deshalb wird zunächst von hormonellen und natürlich von irreversiblen operativen Strategien Abstand genommen und eine meist interdisziplinäre medizinische Betreuung angeboten; bei großem Leidensdruck in der Pubertät kann ab etwa 12 Jahren eine Pubertätsunterdrückung mit Hormon-Antagonisten zum Zeitgewinn sinnvoll sein (Kevan et al. 2016; Hembree et al. 2009). In den meisten westlichen Ländern können irreversible Eingriffe erst ab dem Alter der Volljährigkeit durchgeführt werden. In manchen Ländern bietet man Betroffenen aber die Behandlung zur Pubertätsunterdrückung mit GnRHAnaloga - frühestens allerdings mit 12 Jahren - an. Mit 16 Jahren kann dann mit einer gegengeschlechtlichen Hormontherapie in aufsteigender Dosierung begonnen werden, um die Ausprägung der unerwünschten Geschlechtsmerkmale zu unterdrücken. Die diesbezüglichen Erfahrungen sind durchaus positiv (Kreukels et al. 2011); eine kontinuierliche psychiatrische Re-Evaluation der Diagnose und eine entsprechende psychologische Betreuung und begleitende Psychotherapie sollten aber jedenfalls angeboten werden. Diese Therapieoption sollte auch speziellen Zentren vorbehalten bleiben und die Betroffenen in wissenschaftliche Studien einbezogen werden, um hier weitere verlässliche Informationen gewinnen zu können (Kevan et al. 2016).

\section{Gegengeschlechtliche Hormontherapie}

Nach dem diagnostischen Prozess kann auf Wunsch der Beginn der gegengeschlechtlichen Hormontherapie nach Ausschluss von medizinischen Kontraindikationen erfolgen. Wegen des nach wie vor existenten Stigmas bei Transidentität kann es in der Phase, in der die Person bereits über weite Strecken im gewünschten Geschlecht lebt, zu sozialen Irritationen oder Reaktionen kommen, die ihrerseits zu Stress oder anderen psychischen Reaktionen führen, oder es können in der Realität nicht zutreffende Erwartungen und Vorstellungen der Betroffenen sichtbar werden. Deshalb wird diesen generell vor weiteren irreversiblen Schritten empfohlen, eine etwa 12-monatige „Alltags“-Testphase einzuhalten. Eventuell hier auftretende Probleme und Konflikte können von begleitender psychologischer Therapie behandelt werden (WPATH 2011). 
Manche Betroffene möchten grundsätzlich eine maximale feminisierende bzw. maskulinisierende Hormontherapie, andere wiederum vielleicht nur eine Minimierung der Ausprägung bestehender sekundärer Geschlechtsmerkmale und damit eine geringere hormonelle Dosierung. Die Hormontherapie ist deshalb individuell auf die Wünsche oder sozialen Umstände der Betroffenen und v. a. auf die individuelle Risikosituation abzustimmen. Voraussetzungen für die Initiierung einer potentiell mit irreversiblen Folgen verbundenen Hormontherapie sind eine persistierende, gut dokumentierte Gender-Inkongruenz und die bestehende Einsichtsfähigkeit bezüglich der Behandlung nach ausführlicher Beratung hinsichtlich aller Vorteile und möglicher Nebenwirkungen („Informed Consent“) sowie das Erreichen der Altersgrenze von 18 Jahren (Ausnahmen siehe oben).

Nach Initiierung der Hormontherapie kommt es naturgemäß zu graduellen physischen Veränderungen, wobei diese durchaus individuell variabel sein können und klarerweise auch von der Dosierung, den verwendeten Substanzen sowie der Applikationsart abhängen. Sowohl über potenzielle Nebenwirkungen als auch über die durchschnittliche Dauer bis zum Eintritt des maximal erzielbaren Effektes sollten die Betroffenen ebenfalls vorab informiert werden (Kevan et al. 2016).

Üblicherweise werden in den westlichen Ländern sowohl die Initiierung einer Hormontherapie als auch die - meist langjährige - Überwachung von diesbezüglich spezialisierten Ärztinnen und Ärzten in entsprechenden Zentren durchgeführt, was aber nicht heißen muss, dass nicht auch niedergelassene Ärztinnen und Ärzte mit Kenntnissen auf dem Gebiet der Hormontherapie begleitend tätig werden können. Eine entsprechende Kommunikation mit dem Zentrum ist dabei sicherlich wünschenswert. Die Hormonersatztherapie wird normalerweise lebenslang nach Entfernungen der Gonaden durchgeführt, wobei eine Adjustierung bezüglich der Dosis vom Alter und von anderen Komorbiditäten oder Lebensstiländerungen abhängen kann.

Da es durch den Beginn einer gegengeschlechtlichen Hormontherapie auch zu einer Beeinträchtigung der Fertilität und durch die operative Entfernung der Gonaden zur Infertilität kommt, muss vor Initiierung auf die Möglichkeit des Erhalts der Fertilität durch Kryo-Konservierung der Gonaden hingewiesen werden (Ethics Committee of the American Society for Reproductive Medicine 2015). Da die entsprechenden Optionen jedoch erst in den letzten Jahren entwickelt wurden, gibt es noch sehr wenig wissenschaftliche Daten; die meiste Erfahrung stammt aus Studien zum Erhalt der Fertilität bei malignen Erkrankungen vor einer Chemotherapie oder einer chirurgischen Entfernung der Gonaden. Die wenigen bisher vorlie- 
genden Daten zur psychosozialen Situation von Kindern bei Transidentität lassen keinen Schluss darauf zu, dass durch diese Personen aufgezogene Kinder in irgendeiner Weise beeinträchtigt sind (Chiland et al. 2013). Bei Kindern gleichgeschlechtlicher Partner sind diese Daten wesentlich umfangreicher und zeigen ebenfalls keinerlei Beeinträchtigung (Rupp 2009). Deshalb sollten heute jedenfalls die Möglichkeiten des Fertilitätserhalts mit den betroffenen Personen unbedingt besprochen werden. Die Angebote sollten alle Arten der heute möglichen assistierten Reproduktion beinhalten und die Resultate entsprechender Behandlungen aufgrund der noch nicht ausreichenden Datenlage unbedingt in wissenschaftliche Untersuchungen eingebracht werden.

Auf Basis eines klaren Konsensus aller beteiligten Fachkräfte bezüglich der Kontinuität und Unbeeinflussbarkeit des transidenten Wunsches bzw. der angestrebten Geschlechterrolle ergibt sich nach einer gegengeschlechtlichen Hormontherapie meist dann die Indikation für eine operative Behandlung der Geschlechtsanpassung, die klarerweise ebenfalls nur von entsprechend spezialisierten Zentren durchgeführt werden sollte. Eine adäquate postoperative Nachsorge erfolgt zumeist in diesen Zentren (WPATH 2011; Coleman et al. 2012).

\section{Geschlechtsanpassende Operationen}

Zunächst ist festzuhalten, dass manche transidente Personen durchaus auch ohne Operation(en) mit ihrem Status zufrieden sind. Bei anderen wiederum steht nach der hormonellen Einstellung die weitere Geschlechtsangleichung an, die grundsätzlich zunächst aus dem operativen Teil der Entfernung der Organe des bestehenden Geschlechts besteht. Bei einer MzF-Transidentität bedeutet dies die Entfernung von Hoden und Penis, bei FzM die Entfernung von Eierstöcken, Eileiter, Gebärmutter und (teilweise) der Scheide sowie der Brüste mit eventueller MamillenTransplantation. Im nächsten Teil der operativen Geschlechtsanpassung wird versucht, die Organe des anderen Geschlechts nachzubilden, und dies erfolgt heute meist in weiteren separaten Schritten, da gegebenenfalls auch mehrfache operative Eingriffe nötig sind und bei überlangen Operationen die Morbidität zunimmt. Und schließlich sind noch weitere operative Eingriffe wie Liposuktion, Lipo-Filling, Gesichts-, Schilddrüsen-, Stimmbandoder Gesäßoperationen sowie verschiedene plastisch-ästhetische Eingriffe mit kosmetischen Aspekten denkbar.

Bei transidenten Personen kann es grundsätzlich für geplante operative Schritte zu einem Konflikt zwischen dem Prinzip des Rechts auf Selbstbe- 
stimmung und dem Nichtschadensprinzip von Seiten der behandelnden ÄrztInnen kommen, wenn die Voraussetzungen in Form verschiedener Fachgutachten und/oder von zu durchlaufenden Phasen wie z. B. langzeitiger Hormontherapie als Bevormundung durch Medizin und Gesellschaft empfunden werden. Hier ist einerseits zu beachten, dass es mit zunehmender Irreversibilität der (operativen) Eingriffe eine zunehmende Verantwortung der durchführenden Operateure gibt. Medizinethisch bedarf jeder operative Eingriff einer Indikation, die dadurch gegeben ist, dass der Eingriff in der Lage ist, das umfassende Wohl der betroffenen Person oder zumindest bestimmte Aspekte davon wirksam zu fördern. Auf der anderen Seite sollten alle diagnostischen und gutachterlichen Vorkehrungen eine erfolgreiche Transition ins andere Geschlecht ermöglichen und gleichzeitig Betroffene vor unüberlegten und vorschnellen Interventionen schützen, die diese später vielleicht bereuen. Dies setzt eine umfangreiche Auseinandersetzung mit dem Thema voraus und sollte keine Bevormundung auf der Basis überkommener Anschauungen sein. Darüber hinaus kann das Recht auf Selbstbestimmung bekanntermaßen nur erfolgreich ausgeübt werden, wenn Personen umfassend aufgeklärt sind. Es braucht in diesem Sinn ein umfassendes Wissen der Betroffenen über die Bedingungen einer erfolgreichen Transition, das auf verständliche Weise vermittelt werden muss. Erfolgreiche Selbstbestimmung braucht aber auch die Möglichkeit von „Selbstaufklärung“ und damit Angebote für Betroffene, sich ihrer eigenen Wünsche kritisch zu vergewissern.

Seit 2011 liegen die "Standards of Care“ zur Diagnostik und Behandlung von Transidentität der WPATH in der erweiterten 7. Version vor (www.wpath.org). Die Indikation bei persistenter und dokumentierter Transidentität zur operativen Genitalangleichung sollte laut diesen Empfehlungen von zwei unabhängigen und auf dem Gebiet erfahrenen Gutachtern bescheinigt werden. In den meisten Ländern ist auch die Volljährigkeit der betreffenden Person notwendig; eine obere Altersgrenze besteht hingegen üblicherweise nicht. Speziell für Genitaloperationen wird nach wie vor eine etwa 12-monatige Erfahrung mit einer gegengeschlechtlichen Hormontherapie (falls nicht kontraindiziert) empfohlen, bevor irreversible operative Verfahren angewendet werden (www.wpath.org). Üblicherweise wird - wie in Österreich - auch eine Kostenübernahme-Erklärung der gesetzlichen Krankenversicherungen gefordert. Die ausführliche präoperative Aufklärung mit ausreichendem Abstand zur Operation und eine realistische Einschätzung der Ergebnisse und v.a. die eingehende Erörterung der signifikanten Komplikationsraten mit den Betroffenen gehören heute klarerweise zum Standard in der Informationsübermittlung. Langzeitstudien haben jedenfalls die unzweifelhaften Vorteile von operativen Anglei- 
chungen an das gewünschte Geschlecht in Bezug auf das subjektive Wohlbefinden, kosmetische Ergebnisse und sexuelle Funktionen nachgewiesen (Gijs et al. 2007; Klein et al. 2009).

\section{Medizinischer und gesellschaftlicher Umgang mit Transidentität}

Grundsätzlich sollte heute jeglicher Form der Diskriminierung von transidenten Personen in allen medizinischen, aber auch sonstigen Lebensbereichen wie Schule, Arbeitsleben, Gesellschaft usw. entgegengewirkt werden und zur Vermeidung von Ausgrenzung bereits im Erziehungsbereich, v. a. aber bei der Ausbildung von medizinischem Personal, eine entsprechende Information und Aufklärung erfolgen.

Betroffene Personen sollten einen Anspruch auf umfassende interdisziplinäre Beratung und Betreuung sowie Zugang zu medizinischen Maßnahmen wie Hormontherapie und operativen Möglichkeiten der Geschlechtsumwandlung - idealerweise in entsprechenden Kompetenzzentren - haben. Die Kosten sollten als Behandlungskosten von den gesetzlichen Versicherungen übernommen werden. Die derzeit meist praktizierte Zweistufigkeit der Behandlung, die nach einer hormonellen Behandlung und vor operativen Eingriffen eine Bewährungsprobe im Alltag vorsieht, ist medizinisch vernünftig und wird von einschlägigen Experten befürwortet (WPATH 2011). Eine Geschlechtsumwandlung bei minderjährigen Personen vor Erreichen der Einwilligungsfähigkeit bzw. Volljährigkeit ist jedenfalls aus ethischem Blickwinkel schwierig, da sich hier medizinisch oftmals noch Änderungen der Selbsteinschätzung ergeben können und auch operative Ergebnisse oft suboptimal sind.

Juristisch ist in vielen europäischen Ländern die Situation für transidente Personen ebenfalls noch nicht optimal. Man denke dabei etwa an die Notwendigkeit einer Abfrage des Geschlechts bei alltäglichen Registrierungen usw., bei der transidente Personen Schwierigkeiten haben können. Namensänderungen sollten darüber hinaus nicht von einer Eintragung des Geschlechts im Geburtenbuch und schon gar nicht von einer operativen Behandlung abhängig gemacht werden, sondern die Umschreibung des Geschlechts sollte ausschließlich auf der Grundlage des persönlichen Geschlechtsempfindens erfolgen. 


\section{Literatur}

Chiland, Colette/Clouet, Anne Marie/Golse, Bernard/Guinot, Michèle/Wolf, JeanPhilippe: A new type of family: Transmen as fathers thanks to donor sperm insemination. A 12-year follow-up exploratory study of their children, in: Neuropsychiatrie de l'Enfance et de l'Adolescence 61/6 (2013) 365-370.

Coleman, Eli/Bockting, Walter/Botzer, Marsha et al.: Standards of care for the health of transsexual, transgender, and gender-nonconforming people, version 7, in: International Journal of Transgender 13/4 (2012) 165-232.

Condat, Agnès/Mendes, Nicolas/Drouineaud, Véronique/Gründler, Nouria/ Lagrange, Chrystelle/Chiland, Colette/Wolf, Jean-Philippe/Ansermet, François/ Cohen, David: Biotechnologies that empower transgender persons to self-actualize as individuals, partners, spouses, and parents are defining new ways to conceive a child. Psychological considerations and ethical issues, in: Philosophy, Ethics, and Humanities in Medicine 13 (2018) 1-11.

Ethics Committee of the American Society for Reproductive Medicine: Access to fertility services by transgender persons: an Ethics Committee opinion, in: Fertil Steril 104 (2015) 1111-1115.

Gijs, Luk/Brewaeys, Anne: Surgical treatment of gender dysphoria in adults and adolescents: Recent developments, effectiveness, and challenges, in: Annual Review of Sex Research 18/1 (2007) 178-224.

Hembree, Wylie C./Cohen-Kettenis, Peggy/Delemarre-van de Waal, Henriette A./ Gooren, Louis J./Meyer, Walter J./Spack, Norman P./Tangpricha, Vin/Montori, Victor M.: Endocrine treatment of transsexual persons. An Endocrine Society clinical practice guideline, in: Journal of Clinical Endocrinology \& Metabolism 94/9 (2009) 3132-3154.

Kevan, Wylie/Knudson, Gail/Khan, Sharful Islam/Bonierbale, Mireille/ Watanyusakul, Suporn/Baral, Stefan: Serving transgender people. Clinical care considerations and service delivery models in transgender health, in: Lancet 388/10042 (2016) 401-411.

Klein, Carolin/Gorzalka, Boris B.: Sexual functioning in transsexuals following hormone therapy and genital surgery: A review, in: Journal of Sexual Medicine 6/11 (2009) 2922-2939.

Kreukels, Baudewijntje P./Cohen-Kettenis Peggy T.: Puberty suppression in gender identity disorder: The Amsterdam experience, in: Nature Reviews Endocrinology $7 / 8$ (2011) 466-472.

Kruijver, Frank P. M./Zhou, Jiang-Ning/Pool, Chris W./Hofman, Michel A./ Gooren, Louis J. G./Swaab, Dick F.: Male-to-Female Transsexuals Have Female Neuron Numbers in a Limbic Nucleus, in: Journal of Clinical Endocrinology \& Metabolism 85/5 (2000) 2034-2041.

Kuyper, Lisette: Transgenders in Nederland: prevalentie en attitudes, in: Tijdschrift voor Seksuologie 36/2 (2012) 129-135. 
Meyer zu Hoberge, Sonja: Prävalenz, Inzidenz und Geschlechterverhältnis der Transsexualität anhand der bundesweit getroffenen Entscheidungen nach dem Transsexuellengesetz in der Zeit von 1991 bis 2000, Kiel: 2009 [Inauguraldissertation].

Rupp, Marina (Hg.): Die Lebenssituation von Kindern in gleichgeschlechtlichen Lebenspartnerschaften, Köln: Bundesanzeiger Verlag 2009.

Smith, Elke Stefanie/Junger, Jessica/Derntl, Birgit/Habel, Ute: The transsexual brain. A review of findings on the neural basis of transsexualism, in: Neuroscience and Biobehavioral Reviews 59 (2015) 251-266.

The World Professional Association for Transgender Health (WPATH): Standards of Care for the Health of Transsexual, Transgender and Gender-Nonconforming People, Version 7, in: www.wpath.org [20.03.2020]. 
